\title{
Review Article \\ Responders to Platelet-Rich Plasma in Osteoarthritis: A Technical Analysis
}

\author{
Christophe Milants, ${ }^{1}$ Olivier Bruyère, ${ }^{2,3}$ and Jean-François Kaux ${ }^{1,3}$ \\ ${ }^{1}$ Physical Medicine, Rehabilitation and Sports Traumatology Department, SportS2, FIFA Medical Centre of Excellence, \\ University and University Hospital of Liège, Liège, Belgium \\ ${ }^{2}$ Department of Public Health, Epidemiology and Health Economics, University of Liège, Liège, Belgium \\ ${ }^{3}$ Department of Sports and Rehabilitation Sciences, University of Liège, Liège, Belgium
}

Correspondence should be addressed to Jean-François Kaux; jfkaux@chu.ulg.ac.be

Received 12 November 2016; Revised 15 January 2017; Accepted 30 January 2017; Published 20 August 2017

Academic Editor: Giuseppe Filardo

Copyright (C) 2017 Christophe Milants et al. This is an open access article distributed under the Creative Commons Attribution License, which permits unrestricted use, distribution, and reproduction in any medium, provided the original work is properly cited.

Purpose. To evaluate the similarities and differences between the variety of platelet-rich plasma (PRP) formulations, preparation, and uses to try to determine the best responses for the treatment of knee osteoarthritis. Materials and Methods. A comparison of the outcomes of randomized controlled trials (RCTs) included in the 3 most recent and high-quality meta-analyses to classify the different studies in 2 groups (bad responders group (BRG) and very good responders group (VGRG)). Results and Discussion. From the 19 RCTs analyzed, 7 trials were included in the VGRG and 4 in the BRG. In VGRG, 1 or 2 injections were performed in $4 / 7$ trials, time between injections was 2 to 3 weeks in $4 / 5$ studies with many injections, volume injected varied from 2.5 to $8 \mathrm{~mL}$, and single spinning technique was used in 5/7 studies. PRP classification was Mishra 4B and PAWP2B $\beta$ in 5/7 studies. The use of PRP with leukocytes is only found in the BRG. Conclusion. There is a lack of standardization in PRP preparation technique for knee osteoarthritis. However it appears that the use of a single spinning technique, a platelet concentration lower than 5 times the baseline, and avoidance of leukocytes should be preferred.

\section{Introduction}

Knee osteoarthritis (OA) is one of the major causes of pain and physical disability in older adults. Symptomatic knee OA occurs in approximately $13 \%$ of people who are aged $\geq 60$ years old $[1,2]$. It is a clinical syndrome of joint pain with multifactorial etiopathogenesis that is characterized by the gradual loss of articular cartilage, osteophyte formation, subchondral bone remodeling, and inflammation of the joint [3]. Radiographic knee OA incidence in women $\geq 55$ years was estimated at $2.5 \%$ per year [1].

Different methods are used to alleviate the symptoms of patients with knee OA, including analgesics, physical therapy, exercise prescription, and intra-articular injections, such as corticosteroids injection and hyaluronic acid (HA) $[4,5]$. Owing to the limited lifespan of joint replacements with implant wear and the associated risk for joint revision, conservative treatment modalities are the central focus in the younger and middle-aged population with cartilage damage and $\mathrm{OA}$ of the knee [6].

The management of chondral disease is challenging because of its inherent low healing potential. In fact, the regeneration ability of cartilage is limited due to its isolation from systemic regulation and its lack of vessels and nerves $[7,8]$.

New studies have focused on modern therapeutic methods that stimulate cartilage healing process and improve the damage, including the use of platelet-rich plasma (PRP), an autologous growth factor treatment [4]. PRP is prepared from autologous blood by centrifugation to obtain a highly concentrated sample of platelets, which is four to five times higher than that of normal blood. The platelets undergo degranulation to release growth factors (GFs) that promote angiogenesis, tissue remodeling (bone, skin, muscle, tendon, 
etc.), and wound healing $[9,10]$. More recently, PRP has been used in the management of knee OA. The importance behind using PRP in cartilage tissue engineering field is that PRP is rich in growth factors, including those that promote proliferation of chondrogenic cells and secretion of cartilaginous matrix [11].

Many papers were published on PRP for knee OA, including a lot of randomized controlled trials (RCTs) and different systematic reviews. Due to the mixed results from controlled studies, the clinical efficacy of PRP in the treatment of knee $\mathrm{OA}$ is unclear with shortcomings in the current literature. Indeed, there are variations in the treatment approach including subject, knee and outcomespecific variables, including, but not limited to PRP preparations techniques, platelet count, severity of $\mathrm{OA}$, number of injections, interval/frequency of administration, and a lack of volume standardization [12,13]. In addition, the use of anticoagulants, activating agents, and separation techniques has varied considerably among studies. Campbell et al. published recently a systematic review of meta-analyses, evaluating platelet-rich plasma injection in the treatment of knee joint cartilage degenerative pathology [14]. They included 3 high-quality meta-analyses. Campbell et al. emphasized that there still remain multiple unanswered questions about the best PRP formulation. Different classification of PRP was proposed by various authors to organize and compare results in the literature but remains probably incomplete considering the number of parameters that can characterize PRP [15].

The objective of this study was to evaluate the similarities and differences between the variety of PRP formulations, preparation, and uses of this techniques and to try to determine characteristics of the PRP which tend to give the best result.

\section{Methods}

In November 2015, Campbell et al. published a systematic review of meta-analyses, evaluating platelet-rich plasma injection in the treatment of knee OA [14]. We selected the studies included in the 3 most recent meta-analyses [16-18] on the topics. One RCT was excluded because it was written in Chinese [19]. Two other RCTs were added, after an updated literature search [20,21].

We listed the outcomes that were assessed and reported by each study, including Western Ontario and McMaster Universities Osteoarthritis Index (WOMAC), Visual Analog Scale (VAS), International Knee Documentation Committee (IKDC), and Knee Injury and Osteoarthritis Outcome Score (KOOS).

The minimal clinically important improvement (MCII) was defined to help determining whether an observed difference is clinically important. Tubach et al. promoted in 2012 the use of values of MCII, including knee OA, for different outcome criteria [22]. They determined a value of 15 of 100 for absolute improvement or $20 \%$ for relative improvement. We used Tubach et al's values to classify the different studies in 2 groups depending on the outcomes: bad responders group $(\mathrm{BRG}<\mathrm{MCII})$ and very good responders group (VGRG > 2xMCII).
TABLE 1: The Mishra and PAW classification.

(a) PAW classification system

\begin{tabular}{|c|c|c|c|}
\hline \multirow{4}{*}{\multicolumn{2}{|c|}{ (i) Platelet concentration }} & \multicolumn{2}{|c|}{ P1: $\leq$ baseline } \\
\hline & & \multirow{3}{*}{\multicolumn{2}{|c|}{ 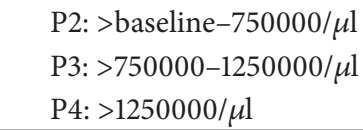 }} \\
\hline & & & \\
\hline & & & \\
\hline \multicolumn{2}{|l|}{ (ii) $\mathrm{X}$} & \multicolumn{2}{|c|}{ Exogenous activation } \\
\hline \multirow{6}{*}{\multicolumn{2}{|c|}{ (iii) White blood cells }} & \multicolumn{2}{|c|}{ (i) Total WBCs } \\
\hline & & \multirow{5}{*}{\multicolumn{2}{|c|}{$\begin{array}{l}\text { A: above baseline } \\
\text { B: } \leq \text { baseline } \\
\text { (ii) Neutrophils: } \\
\text { A: above baseline } \\
\text { B: } \leq \text { baseline }\end{array}$}} \\
\hline & & & \\
\hline & & & \\
\hline & & & \\
\hline & & & \\
\hline \multicolumn{4}{|c|}{ (b) Mishra classification } \\
\hline & WBC & Activation? & $\begin{array}{c}\text { Platelet } \\
\text { concentration }\end{array}$ \\
\hline \multirow{2}{*}{ Type 1} & \multirow{2}{*}{ Increased } & \multirow{2}{*}{ No } & $\mathrm{A}: \geq 5 \mathrm{x}$ \\
\hline & & & $\mathrm{B}:<5 \mathrm{x}$ \\
\hline \multirow{2}{*}{ Type 2} & \multirow{2}{*}{ Increased } & \multirow{2}{*}{ Yes } & $A: \geq 5 x$ \\
\hline & & & $\mathrm{B}:<5 \mathrm{x}$ \\
\hline \multirow{2}{*}{ Type 3} & \multirow{2}{*}{ Minimal or no WBCs } & \multirow{2}{*}{ No } & $A: \geq 5 x$ \\
\hline & & & $\mathrm{B}:<5 \mathrm{x}$ \\
\hline \multirow{2}{*}{ Type 4} & \multirow{2}{*}{ Minimal or no WBCs } & \multirow{2}{*}{ Yes } & $A: \geq 5 x$ \\
\hline & & & $\mathrm{B}:<5 \mathrm{x}$ \\
\hline
\end{tabular}

The full texts were thoroughly read to extract features of the PRP used by the various authors from the 2 groups. Features included the number of injections, time between injections, volume of PRP, centrifugation technique, time after blood puncture before injection, anticoagulation, platelet/erythrocytes or red blood cells (RBC)/leukocytes or white blood cells (WBC) concentration, platelet activation, use of $\mathrm{NaHCO}_{3}$ tampon, and rehabilitation after infiltration.

We contacted all of the authors by e-mail because of insufficient data in the manuscripts.

To classify the PRP of the different studies, we used the Mishra and PAW classifications [23, 24]. These 2 classifications allow the readers to easily know level of platelets concentration, the presence or absence of $\mathrm{WBC}$, and the activation before injection or not (Table 1).

\section{Results}

A total of 19 articles were selected [20, 21, 25-41]. Five authors (Acosta-Olivo, Jang, Patel, Say, and Vaquerizo) out of 19 (26.3\%) gave us more information about the quality of the PRP they used in their study. The outcomes that were assessed and reported by each author are represented in Table 2 . The separation between BRG $(N=4)$ and VGRG $(N=7)$ was made from these values. Eight studies were not included in any group.

In 4 out 7 studies of the VGRG, 1 or 2 injections were given, against 3 in all the studies of the BRG. Time between 


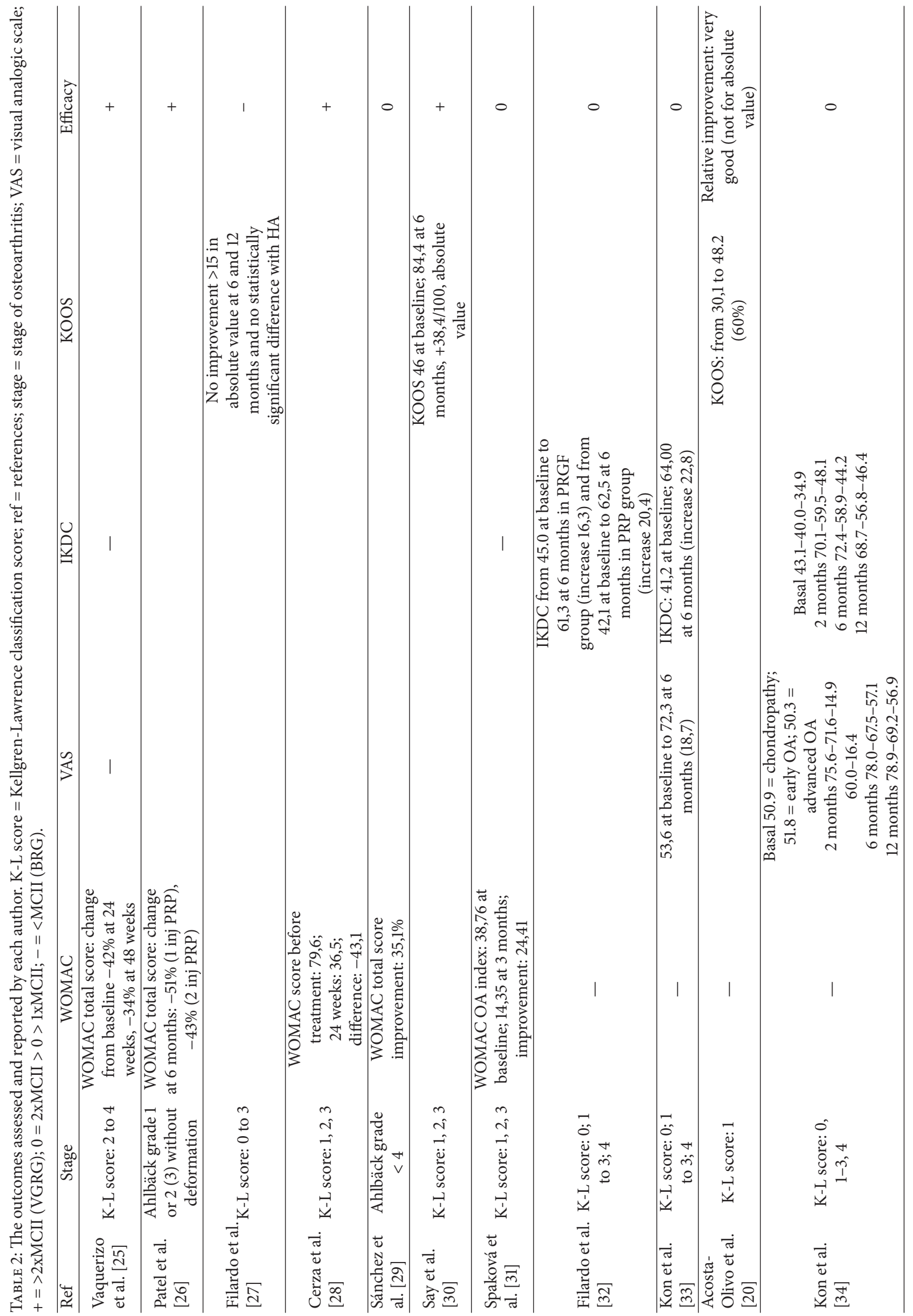




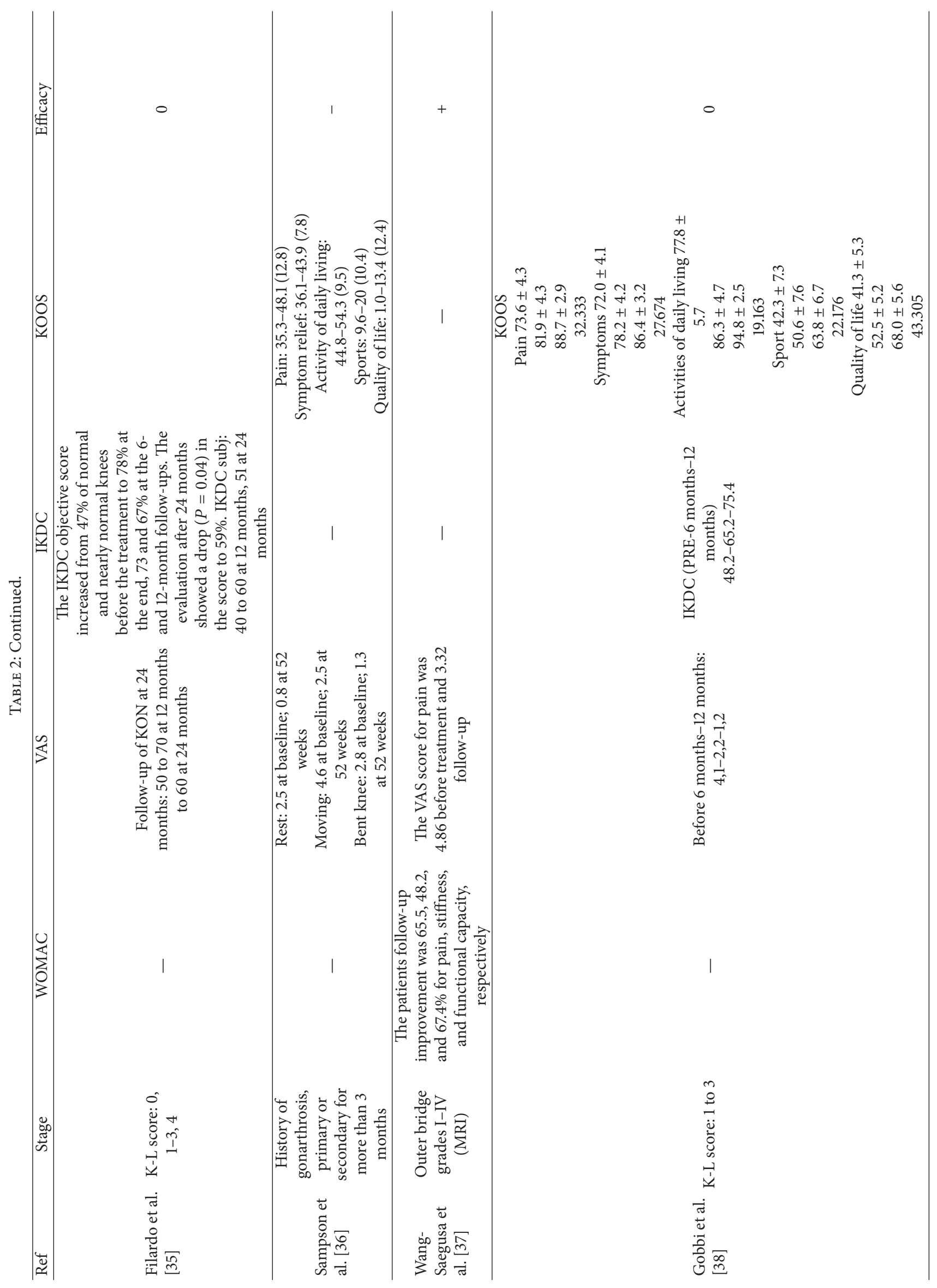




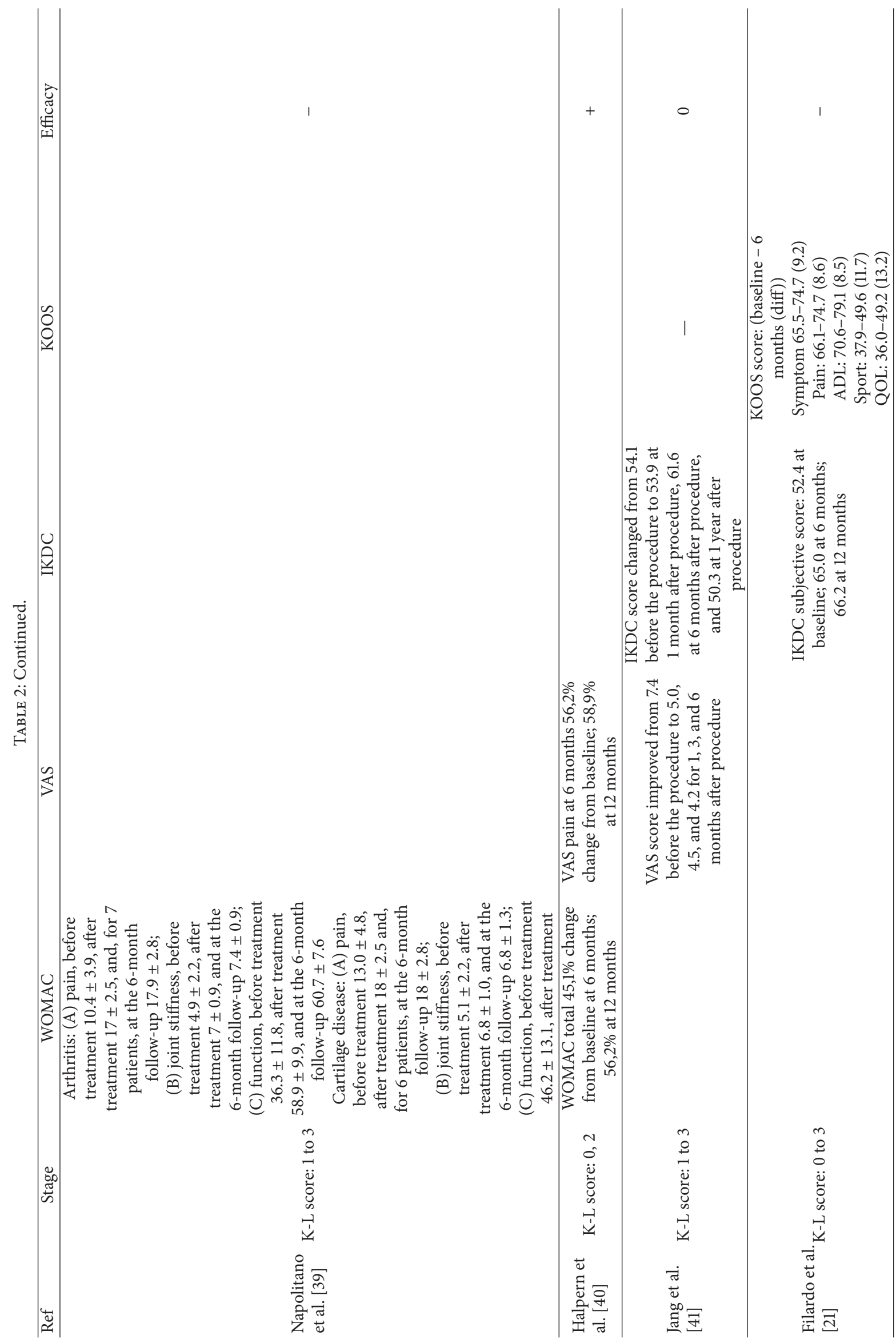


injections tends to be more important in the VGRG (2 to 3 weeks in 4 out of 5 studies with many injections in VGRG in contrast to 1 in BRG). Volume injected varied from $2.5 \mathrm{~mL}$ to $8 \mathrm{~mL}$ in the VGRG. Good results were objectified with the same technique (Endoret), at very different volumes (from 2.5 to $8 \mathrm{~mL}$ ). Centrifugation technique was variable. Single spinning technique was the one used most (6/11; 5/7 VGRG, not mentioned in $2 / 7 ; 1 / 4 \mathrm{BRG}$, not mentioned in $1 / 4$ ) and this technique appears to give better results than double spinning technique. Endoret was used in 3 of 7 good responders and never in bad responders.

Time after blood puncture before injections was less than 2 hours. Freezing technique was used to conserve the PRP units when other injections were scheduled in the following weeks in 2 to 3 out of 11 studies $(N=2 / 4$ in BRG, $N=0$ to $1 / 7$ in VGRG with many injections as it was not mentioned by Wang et al.). In other studies, blood was extracted each time. Anticoagulation, when mentioned, with citrate (CPDA or sodium citrate) was the only used technique $(6 / 11)$ and almost always in VGRG (5/7). Activation with $\mathrm{CaCl}_{2}$ was used in a lot of studies, from the 2 groups (mentioned in $4 / 7$ studies of VGRG and 3/4 studies of BRG).

Platelet concentration was only available in $7 / 11$ studies. The exact value was measured in only 1 out 11 studies and the mean value in only 1 out 11 studies (while it could have been a very important parameter). Other authors compared the platelet concentrations obtained using different preparation techniques [23, 42, 43]. We reported their results to complete the Mishra and PAW classifications. It is interesting to note that almost all studies from the VGRG were Mishra $4 \mathrm{~B}$ and PAW P2B $\beta$. This corresponds to an activated, leukocyte-poor PRP, with a platelet concentration of less than $5 x$ baseline (for Mishra classification), or, more precisely between baseline and 750000 platelets $/ \mu \mathrm{L}$ (for PAW classification) $[23,24]$.

The exact count of RBC and the use of $\mathrm{NaHCO}_{3}$ were never available. The use of PRP with WBC is only found in the BRG. There was no standardized protocol of rehabilitation after infiltration. Most of the time $(N=6 / 11,12 / 19)$, relative rest, analgesics, no nonsteroidal anti-inflammatory drugs (NSAIDs), and light activity were recommended, when mentioned.

\section{Discussion}

We observed an important variability of the PRP preparation technique and a major lack of standardization. Furthermore, a lot of information is missing. However, single spinning technique tends to give good results, more precisely the use an activated, leukocyte-poor PRP with a platelet concentration of less than $5 \mathrm{x}$ baseline. The use of $\mathrm{CaCl}_{2}$ and citrate is frequent. Volume of PRP is inconstant. Leukocyte-rich PRP is only used in the BRG.

Meheux et al. tried to determine similarities and differences in outcomes based on the PRP formulations used in 6 studies [13]. All but one study used leukocyte-poor PRP and showed good outcomes, which did not help comparing the effects of leukocyte-rich PRP versus leukocyte-poor PRP.

We aimed to compare the different formulations of PRPs, after selecting the good and the bad responders, including other features and studies than Meheux et al. November 2012, Tubach et al. promoted the use of values of minimal clinically important improvement (MCII) in reporting the results of trials of different rheumatic diseases, including knee OA, with pain, patient global assessment, physical function, or physician global assessment used as outcome criteria [22]. They determined a value of 15 of 100 for absolute improvement or $20 \%$ for relative improvement.

Only 4 trials did not reach this score, we considered them as bad responders. All of the other studies reached this cutoff score $(N=15 / 19)$. To be more discriminant and to select the studies which results were really the best, we decided to consider as very good responders the trials whose results reached twice this score (Table 3 ). Seven trials were included in this group.

After comparing the results of our analysis, we could propose that the following criteria should be applied:

(i) To focus more on PRP preparation technique than on volume injected, single spinning technique appears attractive, such as PRGF-Endoret by BTI, which is used in 3 of 6 studies from VGRG. It consists of a simple and rapid protocol, with a single spinning approach during 8 minutes at $1800 \mathrm{rpm}$. The PRGF obtained by this technique should not contain WBCs and was first defined by Anitua in 1999 [44]. However, this technique appears to be technically imprecise and may lead to irreproducible results because of the pipetting technique, which is operator-dependent and poses a risk of a low platelet collection efficiency, as platelets and leukocytes are found together in the intermediate layer after low spin centrifugation [15]. Filardo et al. compared safety and efficacy of PRP obtained after single (PRGF) or double (PRP) centrifugation [32]. A significant clinical improvement was obtained in the 2 groups, although PRP injections produced more pain and swelling. Methodological changes and lack of reproducible protocol were emphasized by Anitua et al. [45]. Lack of standardization of PRP preparation could have led to changing results in the literature [23]. The only way to have a reproducible PRP is to use an apheresis machine [46]. In addition to this, its use decreases the potential risk of contamination and the unavoidable variability of manual techniques.

(ii) A platelet concentration 3 to 4 times that of whole blood [46] is recommended for treatment of tendinopathies with PRP. Similar concentrations appear to be recommended for osteoarthritis in view of our results. As it was pointed by Delong et al., this most basic measurement is still lacking in the recent publications [23].

(iii) The presence of WBC is still much debated [23, 32, 44, 47]. Braun et al. showed that using PRP rich in leukocytes and RBC resulted in significant synoviocytes cell death and proinflammatory mediator productions [48]. Some authors prefer avoiding WBC in PRP because of the potential deleterious effect of protease and reactive oxygen released from WBC. Others promote the preventive effect of cytokines and enzymes against infection [32]. A preclinical study highlighted that, in a normal equine tendon, leukocyteriche PRP contributes to inflammatory cytokine production, even if isolated values of IL-1B and TNF- $\alpha$ were increased compared to a leukocyte-poor PRP [47]. In our opinion, 


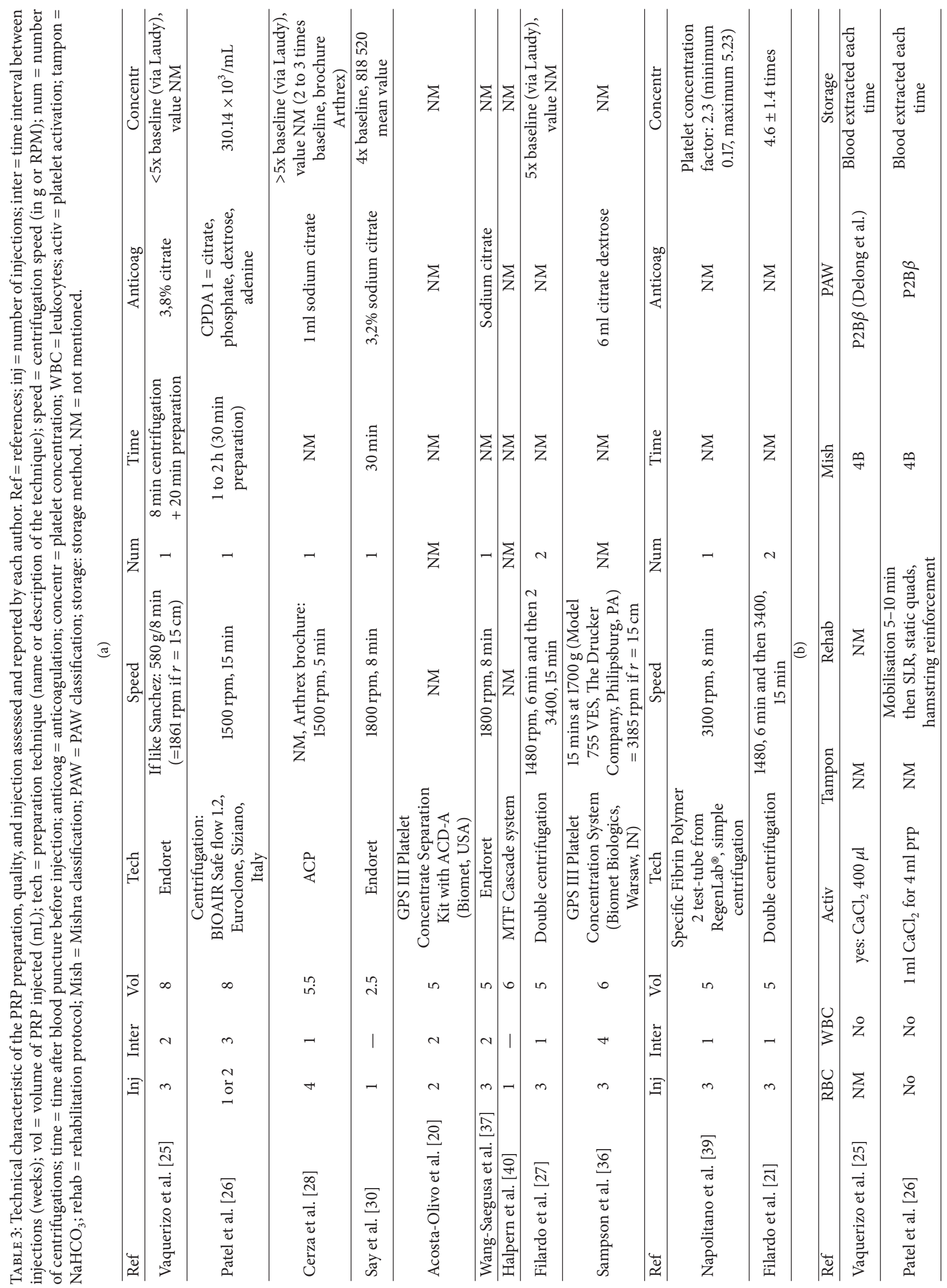




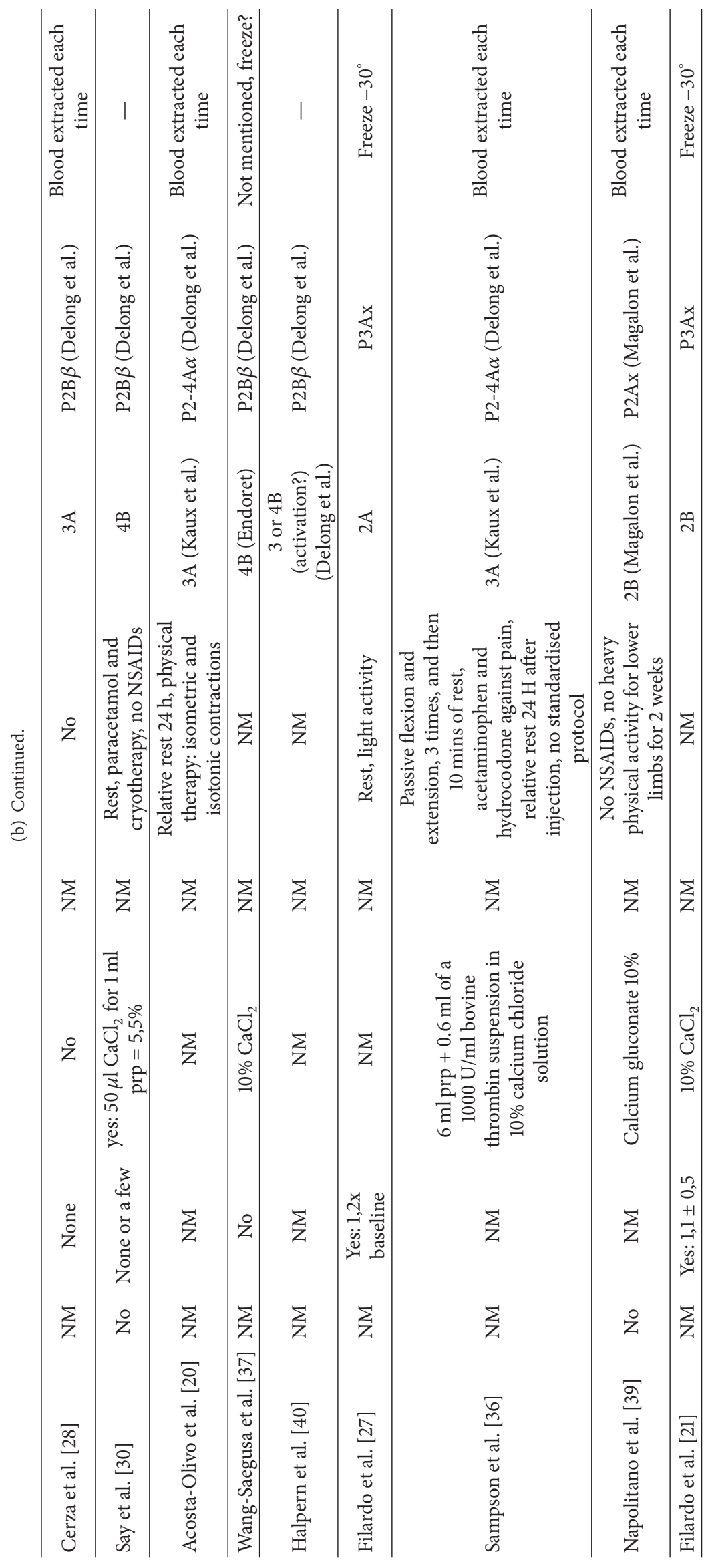


RBC and WBC should be avoided in PRP preparations. Our opinion is not shared by all. A clinical study compared the intra-articular injection of PRGF, which is a PRP obtained after a single centrifugation and which contains no WBC versus PRP obtained after double centrifugation which contain WBC. Both treatment groups presented a similar statistically significant improvement even if the comparative analysis showed more swelling and pain after PRP injection [32]. Furthermore, Mariani et al. showed no upregulation of proinflammatory mediators after leukocyte-rich injection for knee OA in synovial fluid or plasma [49].

(iv) As for tendinopathy treatment, it is necessary to limit the number of injections to avoid side effects and increase in the price of the treatment [50]. Few authors compared the effect of one versus multiple PRP injection for knee OA. Discordant results are available in the literature. Patel et al. compared the effect of a single injection to 2 injections of PRP 3 weeks apart. Short-term results ( 6 weeks) were similar between the two groups. A longer follow-up ( 3 to 6 months) showed that the effect tends to taper over time in the two groups [26]. More recent studies demonstrated beneficial effects of multiple injections. Görmeli et al. showed better results after 3 injections against a single injection for early knee OA after 6 months. For advanced OA, no difference was found [51]. In our daily practice, we realize a total number of 2 injections, as suggested by Kavadar et al. who compared the effects of a single injection, 2 injections, or 3 injections of PRP in moderate knee OA [52]. Future comparative multicentric RCTs on this topic with long term follow-up period should be realized to bring out the most effective injection number.

(v) Injection interval is of 2 to 3 weeks.

(vi) Freezing technique could lead to damage of the PRP product [45]. Sonker and Dubey showed a steep fall in growth factor levels after 5 days of storage after a preparation applying the double freeze thaw technique for the growth factor release [53]. This appears confirmed by our study, because only studies from the BRG (2/4) used this technique to preserve the PRP for further injections. Even if a good clinical efficacy is obtained in studies with PRP stored by freezing [32], it is possible that even better results were obtained with nonfrozen PRP. Note that one author of the VGRG did not mention whether this technique was used or not [37]. The use of an anticoagulant is necessary, citrate is recommended as it preserves platelet reactivity, compared to EDTA and heparin [46]. No difference appears between sodium citrate and citrate dextrose [54].

Even if some authors gave us an answer, some features were not available, because they were not measured and are missing in our comparison table. Platelet concentration, presence or absence of red or white blood cells and its concentration, and use of tampon $\mathrm{NaHCO}_{3}$ were rarely, if ever mentioned. This information should be mentioned in future studies to help determine which PRPs tend to give the best results for osteoarthritis, preferably using a peer reviewed classification system, such as PAW and Mishra classification.

In the literature, other recommendations are described. PRP should be prepared as soon as possible after blood collection, to avoid undesired platelet activation [46]. It is recommended activating platelets by adding $\mathrm{CaCl}_{2}$ [43] and a basic substance like $\mathrm{NaHCO}_{3}$ to get a $\mathrm{pH}$ greater than 8.0 that stimulates platelet activation [46]. Double freeze thaw technique was compared to $\mathrm{CaCl}_{2}$ as a growth factor concentration technique [53]. Levels of PDGF-AB and PDGF-BB were significantly higher with $\mathrm{CaCl}_{2}$ technique whereas there was a nonsignificant trend to higher levels of TGF- $\beta 1$ and IGF- 1 with double-thaw technique. The exact platelet count and $\mathrm{RBC}$ and $\mathrm{WBC}$ count are still unknown. The potential effect of the volume of PRP also has to be considered [55].

A standardization of PRP preparation technique and the use of a reproducible technique would be of great help to interpret and compare the results of the numerous studies, to secondarily bring out the characteristics of the PRP which gives the best results. This current lack and important variability of PRP make this task in time confusing. The degree of knee OA also influences the efficacy of PRP injection and duration of symptoms relief. Better results are achieved in young patients with a little cartilage degeneration [14, 17, 32]. Kon et al. compared the efficacy of PRP injection in patients affected by cartilage degenerative lesions and early and severe knee OA. Better results were achieved in younger patients with a low degree of cartilage degeneration [33].

Limitations of our study are the following. We did not perform a new systematic review of the literature, because many recent meta-analyses are available. We did not compare the studies' methodology, which is very miscellaneous. We arbitrarily decided to be more discriminant in using the cutoff score of twice the MCII value promoted by Tubach et al. to define the very good responders.

\section{Conclusion}

There is a lack of standardization in PRP preparation technique. Our study helped identify features of PRP recommended for knee OA treatment, such as the use of a single spinning technique, a platelet concentration lower than 5 times the baseline (from 3 to 4), and avoiding RBC and WBC. We recommend leveraging this information about PRP for future studies.

\section{Conflicts of Interest}

The authors declare that there is no conflict of interests regarding the publication of this paper.

\section{Acknowledgments}

The authors would like to thank Audrey Collinge for her valuable contribution to this work.

\section{References}

[1] C. Cooper, S. Snow, T. E. McAlindon et al., "Risk factors for the incidence and progression of radiographic knee osteoarthritis," Arthritis \& Rheumatism, vol. 43, no. 5, pp. 995-1000, 2000.

[2] D. T. Felson and M. C. Nevitt, "Epidemiologic studies for osteoarthritis: new versus conventional study design approaches," 
Rheumatic Disease Clinics of North America, vol. 30, no. 4, pp. 783-797, 2004.

[3] E. Ayhan, H. Kesmezacar, and I. Akgun, "Intraarticular injections (corticosteroid, hyaluronic acid, platelet rich plasma) for the knee osteoarthritis," World Journal of Orthopaedics, vol. 5, no. 3, pp. 351-361, 2014.

[4] R. Mascarenhas, B. M. Saltzman, L. A. Fortier, and B. J. Cole, "Role of platelet-rich plasma in articular cartilage injury and disease," The Journal of Knee Surgery, vol. 28, no. 1, pp. 3-10, 2015.

[5] O. Bruyère, C. Cooper, J.-P. Pelletier et al., "An algorithm recommendation for the management of knee osteoarthritis in Europe and internationally: a report from a task force of the european society for clinical and economic aspects of osteoporosis and osteoarthritis (ESCEO)," Seminars in Arthritis and Rheumatism, vol. 44, no. 3, pp. 253-263, 2014.

[6] A. J. Carr, O. Robertsson, S. Graves et al., "Knee replacement," The Lancet, vol. 379, no. 9823, pp. 1331-1340, 2012.

[7] D. J. Hunter, “Osteoarthritis," BMJ, vol. 332, no. 7542, pp. 639642, 2006.

[8] J. Martel-Pelletier, C. Boileau, J.-P. Pelletier, and P. J. Roughley, "Cartilage in normal and osteoarthritis conditions," Best Practice and Research: Clinical Rheumatology, vol. 22, no. 2, pp. 351384, 2008.

[9] F. Smets, J. Croisier, B. Forthomme, J. Crielaard, and J. Kaux, "Applications cliniques du plasma riche en plaquettes (PRP) dans les lésions tendineuses: revue de la littérature," Science \& Sports, vol. 27, no. 3, pp. 141-153, 2012.

[10] J.-F. Kaux, P. Drion, J. L. Croisier, and J. M. Crielaard, "Tendinopathies and platelet-rich plasma (PRP): from preclinical experiments to therapeutic use," Journal of Stem Cells and Regenerative Medicine, vol. 11, no. 1, pp. P7-P17, 2015.

[11] A. Kabiri, B. Hashemibeni, A. Pourazar, M. Mardani, E. Esfandiari, and A. Esmaeili, "Platelet-rich plasma application in chondrogenesis," Advanced Biomedical Research, vol. 3, no. 1, article 138, 2014.

[12] R. T. Nguyen, J. Borg-Stein, and K. McInnis, "Applications of platelet-rich plasma in musculoskeletal and sports medicine: an evidence-based approach," $P M$ and $R$, vol. 3, no. 3, pp. 226-250, 2011.

[13] C. J. Meheux, P. C. McCulloch, D. M. Lintner, K. E. Varner, and J. D. Harris, "Efficacy of intra-articular platelet-rich plasma injections in knee osteoarthritis: a systematic review," Arthroscopy-Journal of Arthroscopic and Related Surgery, vol. 32, no. 3, pp. 495-505, 2016.

[14] K. A. Campbell, B. M. Saltzman, and R. Mascarenhas, "Does intra-articular platelet-rich plasma injection provide clinically superior outcomes compared with other therapies in the treatment of knee osteoarthritis? A systematic review of overlapping meta-analyses," Arthroscopy, vol. 31, no. 11, pp. 2213-2221, 2015.

[15] D. M. Dohan Ehrenfest, L. Rasmusson, and T. Albrektsson, "Classification of platelet concentrates: from pure platelet-rich plasma (P-PRP) to leucocyte- and platelet-rich fibrin (L-PRF)," Trends in Biotechnology, vol. 27, no. 3, pp. 158-167, 2009.

[16] A. B. M. Laudy, E. W. P. Bakker, M. Rekers, and M. H. Moen, "Efficacy of platelet-rich plasma injections in osteoarthritis of the knee: a systematic review and meta-analysis," British Journal of Sports Medicine, vol. 49, no. 10, pp. 657-672, 2015.

[17] K.-V. Chang, C.-Y. Hung, F. Aliwarga, T.-G. Wang, D.-S. Han, and W.-S. Chen, "Comparative effectiveness of platelet-rich plasma injections for treating knee joint cartilage degenerative pathology: a systematic review and meta-analysis," Archives of Physical Medicine and Rehabilitation, vol. 95, no. 3, pp. 562-575, 2014.

[18] A. Khoshbin, T. Leroux, D. Wasserstein et al., "The efficacy of platelet-rich plasma in the treatment of symptomatic knee osteoarthritis: a systematic review with quantitative synthesis," Arthroscopy-Journal of Arthroscopic and Related Surgery, vol. 29, no. 12, pp. 2037-2048, 2013.

[19] M. Li, C. Zhang, Z. Ai, T. Yuan, Y. Feng, and W. Jia, “Therapeutic effectiveness of intra-knee-articular injection of plateletrich plasma on knee articular cartilage degeneration," Chinese Journal of Reparative and Reconstructive Surgery, vol. 25, no. 10, pp. 1192-1196, 2011.

[20] C. Acosta-Olivo, F. Esponda-Colmenares, F. Vilchez-Cavazos, J. Lara-Arias, O. Mendoza-Lemus, and T. Ramos-Morales, "Platelet rich plasma versus oral paracetamol for the treatment of early knee osteoarthritis. Preliminar Study," Cirugia y Cirujanos, vol. 82, no. 2, pp. 163-169, 2014.

[21] G. Filardo, B. Di Matteo, A. Di Martino et al., "Platelet-rich plasma intra-articular knee injections show no superiority versus viscosupplementation: a randomized controlled trial," American Journal of Sports Medicine, vol. 43, no. 7, pp. 15751582, 2015.

[22] F. Tubach, P. Ravaud, E. Martin-Mola et al., "Minimum clinically important improvement and patient acceptable symptom state in pain and function in rheumatoid arthritis, ankylosing spondylitis, chronic back pain, hand osteoarthritis, and hip and knee osteoarthritis: results from a prospective multinational study," Arthritis Care and Research, vol. 64, no. 11, pp. 1699-1707, 2012.

[23] J. M. Delong, R. P. Russell, and A. D. Mazzocca, "Platelet-rich plasma: the PAW classification system," Arthroscopy-Journal of Arthroscopic and Related Surgery, vol. 28, no. 7, pp. 998-1009, 2012.

[24] A. Mishra, K. Harmon, J. Woodall, and A. Vieira, "Sports medicine applications of platelet rich plasma," Current Pharmaceutical Biotechnology, vol. 13, no. 7, pp. 1185-1195, 2012.

[25] V. Vaquerizo, M. Á. Plasencia, I. Arribas et al., "Comparison of intra-articular injections of plasma rich in growth factors (PRGF-Endoret) versus durolane hyaluronic acid in the treatment of patients with symptomatic osteoarthritis: a randomized controlled trial," Arthroscopy-Journal of Arthroscopic and Related Surgery, vol. 29, no. 10, pp. 1635-1643, 2013.

[26] S. Patel, M. S. Dhillon, S. Aggarwal, N. Marwaha, and A. Jain, "Treatment with platelet-rich plasma is more effective than placebo for knee osteoarthritis: a prospective, double-blind, randomized trial," American Journal of Sports Medicine, vol. 41, no. 2, pp. 356-364, 2013.

[27] G. Filardo, E. Kon, A. Di Martino et al., "Platelet-rich plasma vs hyaluronic acid to treat knee degenerative pathology: study design and preliminary results of a randomized controlled trial," BMC Musculoskeletal Disorders, vol. 13, article 229, 2012.

[28] F. Cerza, S. Carnì, A. Carcangiu et al., "Comparison between hyaluronic acid and platelet-rich plasma, intra-articular infiltration in the treatment of gonarthrosis," American Journal of Sports Medicine, vol. 40, no. 12, pp. 2822-2827, 2012.

[29] M. Sánchez, N. Fiz, J. Azofra et al., "A randomized clinical trial evaluating plasma rich in growth factors (PRGF-Endoret) versus hyaluronic acid in the short-term treatment of symptomatic knee osteoarthritis," Arthroscopy-Journal of Arthroscopic and Related Surgery, vol. 28, no. 8, pp. 1070-1078, 2012. 
[30] F. Say, D. Gürler, and K. Yener, "Platelet-rich plasma injection is more effective than hyaluronic acid in the treatment of knee osteoarthritis," Acta Chirurgiae Orthopaedicae et Traumatologiae Cechoslovaca, vol. 80, pp. 278-283, 2013.

[31] T. Spaková, J. Rosocha, M. Lacko, D. Harvanová, and A. Gharaibeh, "Treatment of knee joint osteoarthritis with autologous platelet-rich plasma in comparison with hyaluronic acid," American Journal of Physical Medicine \& Rehabilitation, vol. 91, pp. 411-417, 2013.

[32] G. Filardo, E. Kon, M. T. Pereira Ruiz et al., "Platelet-rich plasma intra-articular injections for cartilage degeneration and osteoarthritis: single- versus double-spinning approach," Knee Surgery, Sports Traumatology, Arthroscopy, vol. 20, no. 10, pp. 2082-2091, 2012.

[33] E. Kon, B. Mandelbaum, R. Buda et al., "Platelet-rich plasma intra-articular injection versus hyaluronic acid viscosupplementation as treatments for cartilage pathology: from early degeneration to osteoarthritis," Arthroscopy-Journal of Arthroscopic and Related Surgery, vol. 27, no. 11, pp. 1490-1501, 2011.

[34] E. Kon, R. Buda, G. Filardo et al., "Platelet-rich plasma: intraarticular knee injections produced favorable results on degenerative cartilage lesions," Knee Surgery, Sports Traumatology, Arthroscopy, vol. 18, no. 4, pp. 472-479, 2010.

[35] G. Filardo, E. Kon, R. Buda et al., "Platelet-rich plasma intraarticular knee injections for the treatment of degenerative cartilage lesions and osteoarthritis," Knee Surgery, Sports Traumatology, Arthroscopy, vol. 19, no. 4, pp. 528-535, 2011.

[36] S. Sampson, M. Reed, H. Silvers, M. Meng, and B. Mandelbaum, "Injection of platelet-rich plasma in patients with primary and secondary knee osteoarthritis," American Journal of Physical Medicine \& Rehabilitation, vol. 89, no. 12, pp. 961-969, 2010.

[37] A. Wang-Saegusa, R. Cugat, O. Ares, R. Seijas, X. Cuscó, and M. Garcia-Balletbó, "Infiltration of plasma rich in growth factors for osteoarthritis of the knee short-term effects on function and quality of life," Archives of Orthopaedic and Trauma Surgery, vol. 131, no. 3, pp. 311-317, 2011.

[38] A. Gobbi, G. Karnatzikos, V. Mahajan, and S. Malchira, "Platelet-rich plasma treatment in symptomatic patients with knee osteoarthritis: preliminary results in a group of active patients," Sports Health, vol. 4, no. 2, pp. 162-172, 2012.

[39] M. Napolitano, S. Matera, M. Bossio et al., "Autologous platelet gel for tissue regeneration in degenerative disorders of the knee," Blood Transfusion, vol. 10, no. 1, pp. 72-77, 2012.

[40] B. Halpern, S. Chaudhury, S. A. Rodeo et al., "Clinical and MRI outcomes after platelet-rich plasma treatment for knee osteoarthritis," Clinical Journal of Sport Medicine, vol. 23, no. 3, pp. 238-239, 2013.

[41] S. Jang, J. Kim, and S. Cha, "Platelet-rich plasma (PRP) injections as an effective treatment for early osteoarthritis," European Journal of Orthopaedic Surgery \& Traumatology, vol. 23, no. 5, pp. 573-580, 2013.

[42] J. Magalon, O. Bausset, N. Serratrice et al., "Characterization and comparison of 5 platelet-rich plasma preparations in a single-donor model," Arthroscopy-Journal of Arthroscopic and Related Surgery, vol. 30, no. 5, pp. 629-638, 2014.

[43] J.-F. Kaux, C. Le Goff, J. Renouf et al., "Comparison of the platelet concentrations obtained in platelet-rich plasma (PRP) between the GPS ${ }^{\mathrm{TM}}$ II and GPS ${ }^{\mathrm{TM}}$ III systems," Pathologie Biologie, vol. 59, no. 5, pp. 275-277, 2011.

[44] E. Anitua, "Plasma rich in growth factors: preliminary results of use in the preparation of future sites for implants," International
Journal of Oral and Maxillofacial Implants, vol. 14, no. 4, pp. 529535, 1999.

[45] E. Anitua, M. Sánchez, R. Prado, and G. Orive, "The type of platelet-rich plasma may influence the safety of the approach," Knee Surgery, Sports Traumatology, Arthroscopy, vol. 22, no. 7, pp. 1708-1709, 2014.

[46] J.-F. Kaux, M. Bouvard, C. Lecut et al., "Reflections about the optimisation of the treatment of tendinopathies with PRP," Muscles, Ligaments and Tendons Journal, vol. 5, no. 1, pp. 1-4, 2015.

[47] T. M. McCarrel, T. Minas, and L. A. Fortier, "Optimization of leukocyte concentration in platelet-rich plasma for the treatment of tendinopathy," Journal of Bone and Joint Surgery A, vol. 94, no. 19, pp. el43.1-e143.8, 2012.

[48] H. J. Braun, H. J. Kim, C. R. Chu, and J. L. Dragoo, "The effect of platelet-rich plasma formulations and blood products on human synoviocytes: implications for intra-articular injury and therapy," The American Journal of Sports Medicine, vol. 42, no. 5, pp. 1204-1210, 2014.

[49] E. Mariani, V. Canella, L. Cattini et al., "Leukocyte-rich Plateletrich Plasma injections do not up-modulate intra- articular proinflammatory cytokines in the osteoarthritic knee," PLOS ONE, vol. 11, no. 6, pp. 1-13, 2016.

[50] J.-F. Kaux, V. Libertiaux, J.-L. Croisier, and J.-M. Crielaard, "Platelet-rich plasma (PRP) to treat chronic patellar tendinopathies: comparison of a single versus two closely-timed injections," Muscles, Ligaments and Tendons Journal, vol. 5, no. 4, pp. 297-298, 2015.

[51] G. Görmeli, C. A. Görmeli, B. Ataoglu, C. Çolak, O. Aslantürk, and K. Ertem, "Multiple PRP injections are more effective than single injections and hyaluronic acid in knees with early osteoarthritis: a randomized, double-blind, placebo-controlled trial," Knee Surgery, Sports Traumatology, Arthroscopy, pp. 1-8, 2015.

[52] G. Kavadar, D. T. Demircioglu, M. Y. Celik, and T. Y. Emre, "Effectiveness of platelet-rich plasma in the treatment of moderate knee osteoarthritis: a randomized prospective study," Journal of Physical Therapy Science, vol. 27, no. 12, pp. 3863-3867, 2015.

[53] A. Sonker and A. Dubey, "Determining the effect of preparation and storage: an effort to streamline platelet components as a source of growth factors for clinical application," Transfusion Medicine and Hemotherapy, vol. 42, no. 3, pp. 174-180, 2015.

[54] C. E. Giraldo, M. E. Álvarez, and J. U. Carmona, "Effects of sodium citrate and acid citrate dextrose solutions on cell counts and growth factor release from equine pure-platelet rich plasma and pure-platelet rich gel," BMC Veterinary Research, vol. 11, no. 1, article 60, 2015.

[55] J. U. Carmona, D. L. Ríos, C. López, M. E. Álvarez, J. E. Pérez, and M. E. Bohórquez, "In vitro effects of platelet-rich gel supernatants on histology and chondrocyte apoptosis scores, hyaluronan release and gene expression of equine cartilage explants challenged with lipopolysaccharide," BMC Veterinary Research, vol. 12, no. 1, article 135, 2016. 


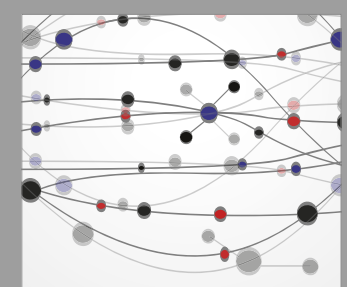

The Scientific World Journal
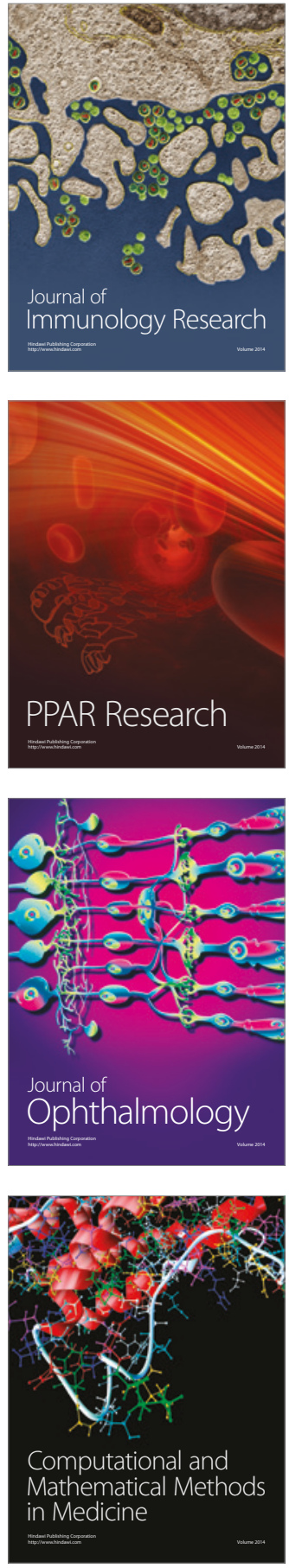

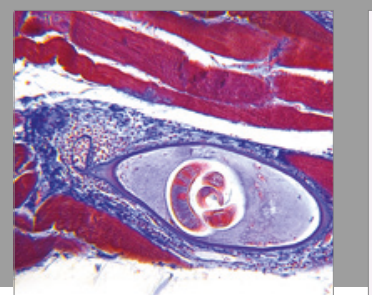

Gastroenterology Research and Practice
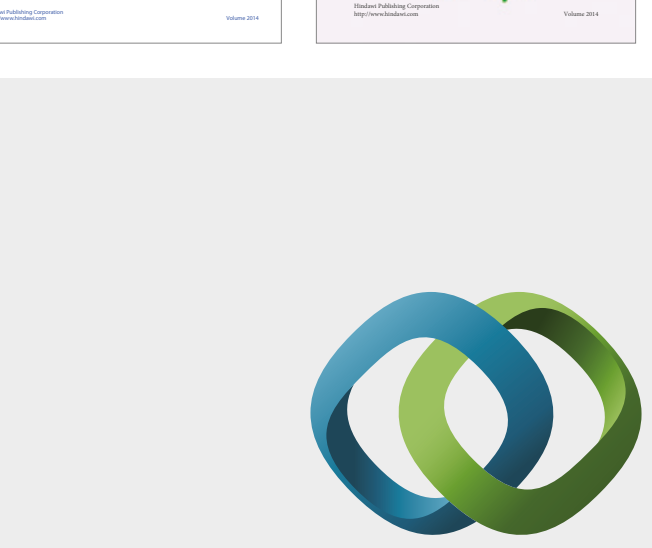

\section{Hindawi}

Submit your manuscripts at

https://www.hindawi.com
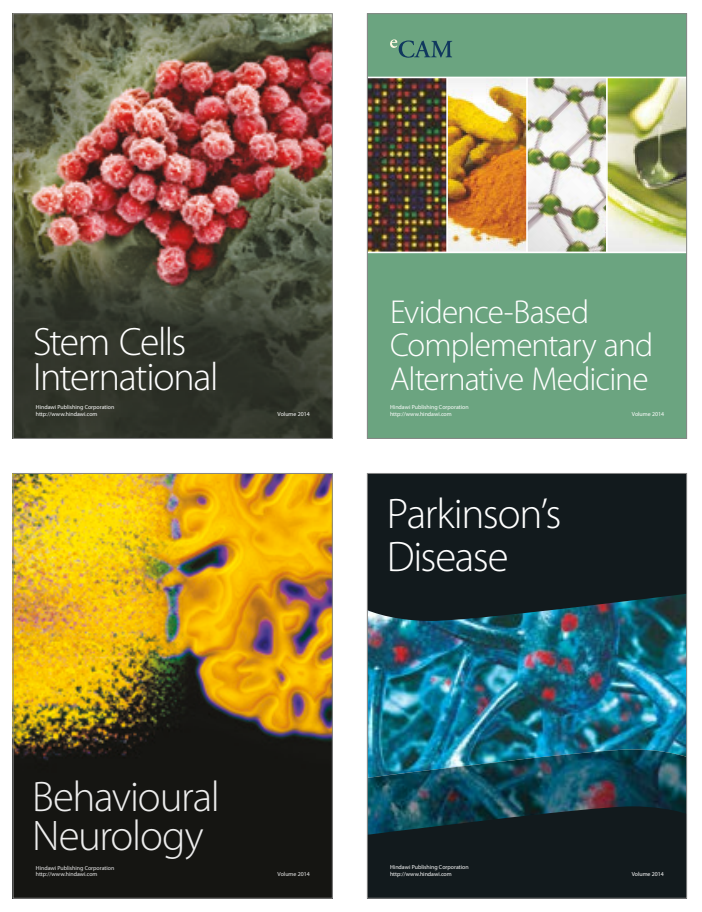
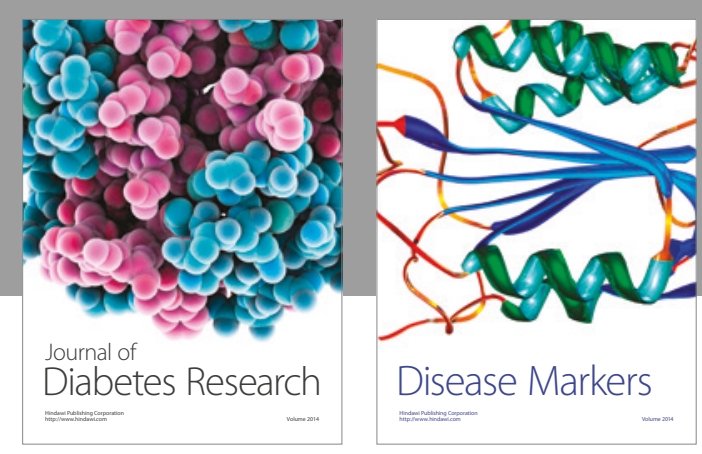

Disease Markers
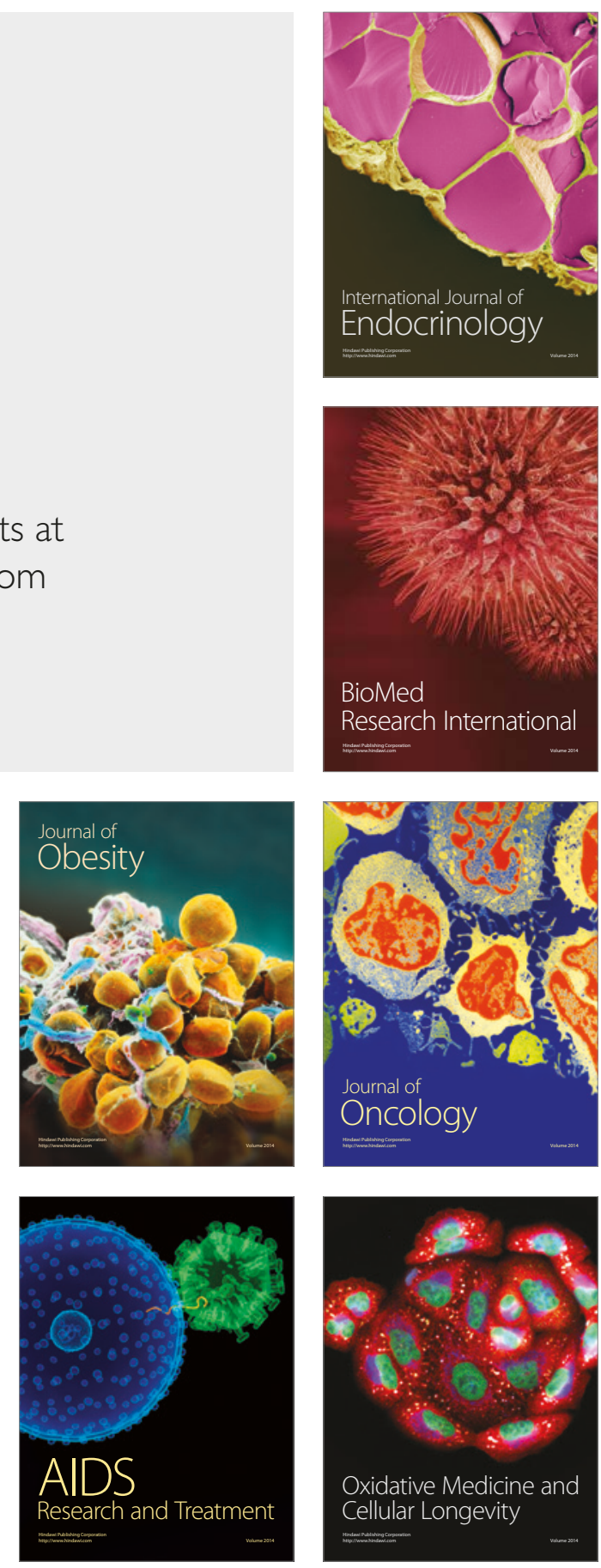